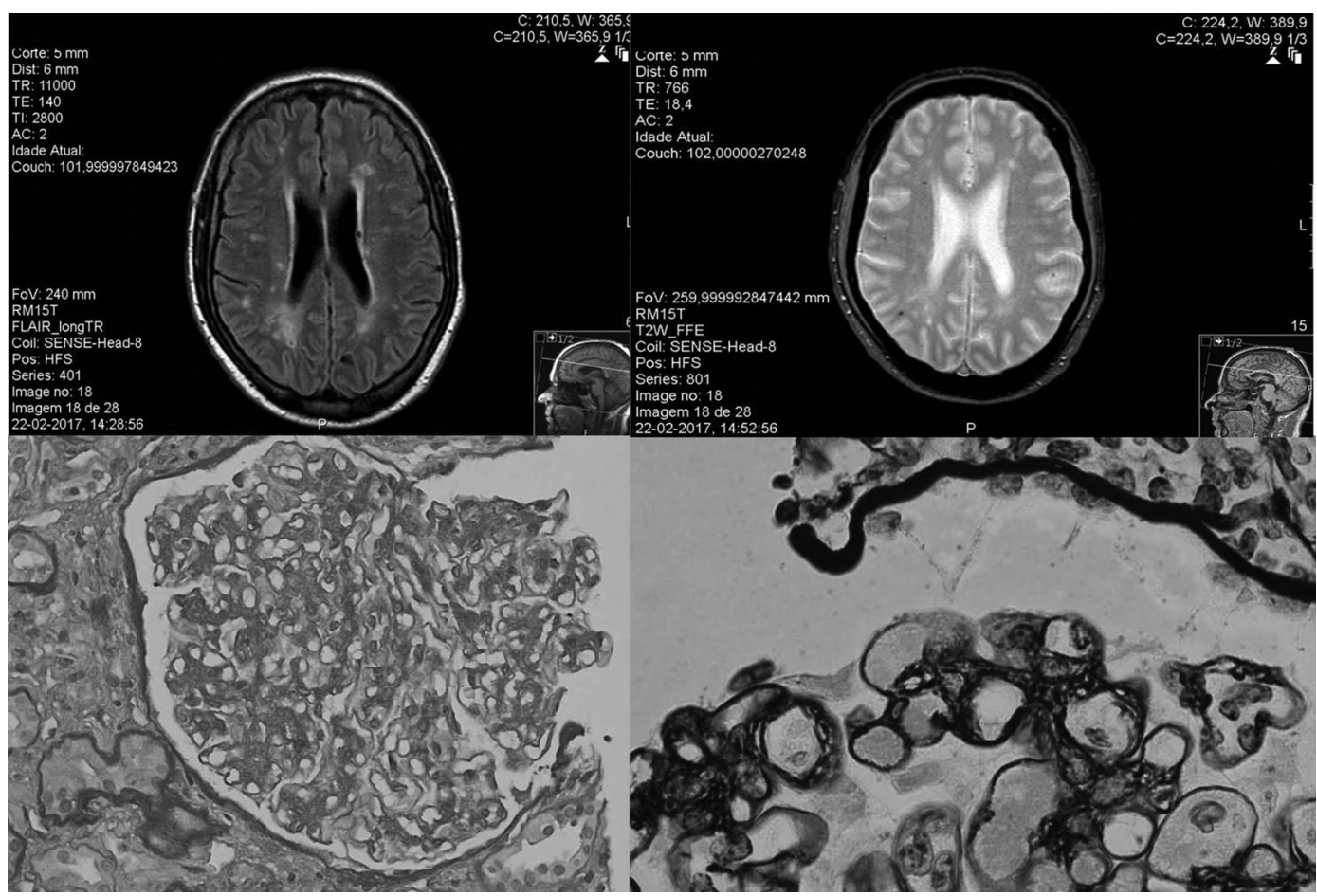

Abstract PS4:76 Figure 1

\section{PS4:77 TWO CASES OF FATAL CATASTROPHIC ANTIPHOSPHOLIPID SYNDROME}

${ }^{1} \mathrm{~L}$ Castelnovo, ${ }^{2} \mathrm{~A}$ Laria, ${ }^{2} \mathrm{~A}$ Lurati, ${ }^{1} \mathrm{~A}$ Tamburello, ${ }^{1} \mathrm{P}$ Faggioli, ${ }^{1} \mathrm{~A}$ Mazzone. ${ }^{1}$ Asst Ovest Milanese, Legnano, Italy; ${ }^{2}$ Asst Ovest Milanese, Magenta, Italy

\subsection{6/lupus-2018-abstract.122}

Introduction Catastrophic antiphospholipid syndrome (CAPS) is a rare and fatal condition secondary to antiphospholipid syndrome (APS) characterised by venous and/or arterial thromboembolism within a short period of time in the presence of positive AP antibodies. Thrombocytopenia is seen in $>60 \%$ of cases. Here we report 2 cases of women with fatal CAPS.Case reports1.A 55-old female affected by APS (excluded overlap SLE), treated initialy with warfarin, due to previous DVT, than apixaban after a new EP episode, was admitted to our Unit for dyspnea: we found a mitral valve injury. After few days she developed a neurologic impairment with evidence of post-ischaemic alterations, likely embolics, and a sensory motor neuropathy. We concluded for probable CAPS and started antibiotics plus high dose corticosteroids, unfractionated heparin and plasma exchange. However, we observed further worsening. The patient quickly succumbed to her illness. Case report 2. A 59-old female affected by SLE with CSN impairment and APS with thrombocytopenia and recurring DVT - EP episodes treated with low dose methylprednisolone, iloprost and heparin (warfarin suspension for severe thrombocytopenia impairment) was admitted to our Unit for appearance of diarrhoea with abdominal pain. Blood tests showed further worsening of platelet count. We started antibiotics with clinical improvement. Taking into account the patient history CT was performed, with evidence of massive arteriovenous and aortic arterial disease severe thrombotic obstruction. Two days later she developed acute pain on the left foot with massive venous thrombosis of the left femoropopliteo axis. We started unfractionated heparin, despite low platelet value, as rescue therapy. Tthe patient progressively worsened with further progression of the arterial occlusions, until death, 3 days later.

Conclusion CAPS can lead to acute multiorgan failure and can be associated with infections or SLE. Early recognition is essential for effective life-saving treatment. Management of these patients is complex, especially in those with thrombocytopenia. Anticoagulation plus steroids plus plasma exchange should are the first line therapy but our two cases show that they are not always applicable and effective. New therapies, such as rituximab and eculizumab, may be options, but time is a fundamental variable.

\section{PS4:78 WOMEN WITH SYSTEMIC LUPUS ERYTHEMATOSUS MORE OFTEN ACHIEVE PREGNANCY, AND HAVE A SHORTER TIME TO PREGNANCY COMPARED TO WOMEN WITH RHEUMATOID ARTHRITIS}

\begin{abstract}
${ }^{1,2} \mathrm{C}$ Gøtestam Skorpen, ${ }^{3} \mathrm{~S}$ Lydersen, ${ }^{4} \mathrm{I} \mathrm{M}$ Gilboe, ${ }^{5} \mathrm{JF}$ Skomsvoll, ${ }^{6,7} \mathrm{~K} \AA \AA$ Salvesen, ${ }^{4} \varnothing$ Palm, ${ }^{5}$ HSS Koksvik, ${ }^{5} \mathrm{~B}$ Jakobsen, ${ }^{1,5} \mathrm{M}$ Wallenius. 'Dept. of Neuromedicine and Movement science, NTNU, Norwegian University of Science and Technology, Trondheim, Norway; ${ }^{2}$ Dept. of Rheumatology, Ålesund hospital, Ålesund, Norway; ${ }^{3}$ Regional Centre for Child and Youth Mental Health and Child Welfare, NTNU, Norwegian University of Science and Technology, Trondheim, Norway; ${ }^{4}$ Dept. of Rheumatology, Oslo University Hospital Rikshospitalet, Oslo, Norway; ${ }^{5}$ Norwegian National Advisory Unit on Pregnancy and Rheumatic Diseases, St Olavs hospital, Trondheim University Hospital, Trondheim, Norway; ${ }^{6}$ Dept. of laboratory medicine, Women and Children Health, NTNU, Norwegian University of Science and Technology, Trondheim, Norway; ${ }^{7}$ Dept. of Obstetrics and Gynaecology, St Olavs hospital, Trondheim University Hospital, Trondheim, Norway
\end{abstract}

10.1136/lupus-2018-abstract.123 